\title{
Partitions chromatiques et convergence des discours médiatiques
}

\author{
L'altérité imaginaire des figures \\ tsiganes à la télévision roumaine
}

Jonathan Larcher ${ }^{1}$

\begin{abstract}
[Résumé] Entre 2007 et 2013, les trois principaux conglomérats de médias roumains produisent des émissions de divertissement et des séries TV où apparaissent des personnages tsiganes. En écho à certains discours médiatiques ou en se réappropriant des formes visuelles produites en dehors de ces industries culturelles, l'œil identitaire de la télévision roumaine mainstream a produit plusieurs régimes visuels de l'altérité. Malgré leur incessante métamorphose, de l'expertisable au grotesque, ils indiquent la relation de pouvoir qu'entretient ces médias avec des figures rejetées à la périphérie.
\end{abstract}

Mots-clés : régime visuel, séries TV, altérité, Roumanie, Tsiganes, Roms

\begin{abstract}
[Abstract] Between 2007 and 2013, the three principal media conglomerates in Romania produced entertainment shows and TV serials in which gypsy characters were represented. In echo with some media discourses or with the reappropriation of visual forms produced outside the cultural industries, the identity eye of Romanian mainstream television produced several visual regimes of alterity. Despite their ceaseless metamorphosis, from the " accountability » to the grotesque, they indicate the relation of power between these media and the characters which are marginalised.
\end{abstract}

Keywords: visual regime, TV series, alterity, Romania, Gypsies, Roma people

\section{Introduction}

Depuis 2007, des figures tsiganes ont fait leur apparition dans des programmes de prime time de la télévision roumaine. Ces personnages masculins (ţigani) et féminins (ţigănci) ont occupé une place centrale dans des séries télévisées et émissions de divertissement produites par les principaux groupes de médias privés roumains. Cette histoire visuelle repose sur deux premières interrogations : quelle figuration de l'identité

1 École des hautes études en sciences sociales. 
ethnique des Romi (Roms) proposent ces images ? Quelle est l'évolution de cette représentation dans les télévisions dites mainstream ? Bien loin de réduire ces figures à de simples « altérités spéculaires » (Stoichiță, 2014, 17), il s'agit au contraire de considérer la complexité de ces formes visuelles et genres télévisuels qui développent à la fois des personnages, des univers, des arcs narratifs et des imageries. Cette entreprise est d'autant plus délicate qu'elle nécessite d'identifier les pratiques et la circulation des images qui ont prévalu à ces représentations de manière explicite, ou de révéler des correspondances inédites entre discours journalistiques et formes visuelles. L'évolution de l'imaginaire lié à ces figures éclaire à la fois une " économie figurative » (Brenez, 1998, 12) propre à une période historique de la télévision roumaine ainsi qu'une conception singulière de l'altérité. Je présenterai dans un premier temps les différentes théories et champs d'études auxquels s'adosse cette analyse. La présentation des trois régimes visuels construits autour de ces figures tsiganes suivra ensuite, selon leur ordre d’apparition dans les programmes télévisés.

\section{Régime(s) visuel(s) de l'altérité}

Une première caractéristique des groupes Romi/Ţigani en Roumanie est leur impossible réduction à une minorité ethnique. En effet, ils ne conçoivent pas leur propre identité dans une opposition binaire aux Gadje (« les non tsiganes »), pas plus qu'ils ne se considèrent comme une entité unique. Ce constat est valable pour la Roumanie (Olivera, 2009) comme pour l'ensemble de l'Europe (Piasere, 2004). La diversité historique, anthropologique et linguistique des groupes de familles romi en Roumanie interdit également de voir dans les groupes socio-professionnels présents depuis quelques années à la télévision des représentants de la dite " ethnie Rom ». Dès lors, parler de la représentation de la minorité tsigane, en tant qu'entité, par ces contenus télévisuels serait une première distorsion.

Dans ce contexte, l'analyse des représentations télévisuelles s'intègre dans une ethnographie de la circulation des médias au sein d'une communauté de Ţigani, constituée en partie de familles de musiciens. L'attention portée à ces logiques de circulation montre à la fois l'instrumentalisation des malentendus dans la constitution de l'identité de ce groupe de Romi/Tigani et la construction d'un régime visuel de l'Altérité qui associe hiérarchie culturelle et ordre national, représentations visuelles et discours sur ces représentations.

Dans les limites imparties ici, je me concentrerai sur les essentialismes et particularismes identitaires proposés par ces représentations visuelles en analysant d'abord les formes narratives et plastiques qui ont contribué à la formation des marqueurs ethniques dans ces contenus de divertissement. Une analyse intertextuelle sera ensuite conduite, sur le contexte de ces productions médiatiques, avec une attention particulière portée à la matrice la plus déterminante dans la formation de chacun de ces régimes visuels. 
Le corpus d'émissions étudiées se constitue de six séries télévisés Inimă de ţigan (Cour de tsigane, Media Pro, 2007-2008), Regina (La Reine, Media Pro, 2008-2009), State de România (State de Roumanie, Media Pro, 2009-2010), Moştenirea (L’Héritage, Media Pro, 2010-2011), Iubiri secrete (Les Amours secrets, ProSiebenSat.1, 2011-...), Spitalul de demenţă (L'Hôpital de folie, Media Pro, 2012-2013) ; et de deux émissions de divertissement Serviciul Român de Comedie (Le Service roumain de Comédie, Media Pro, 20092010), În Puii Mei (Dans mes poussins, Intact, 2009-2011). Ces émissions ont été diffusées entre 2007 et 2013 sur les chaînes des trois principaux groupes privés en termes d'audience et de recettes publicitaires en Roumanie : MediaPro (Acasă TV, Pro TV), Intact (Antena 1) et ProSiebenSat.1 (Prima TV).

\section{D'un essentialisme ethnique aux partitions chromatiques}

Au cours des années 1990, diverses situations politiques et médiatiques ont mis au premier plan des familles romi dans les médias d'information en Roumanie. Avec ses projets d'entrée dans l'Union européenne, la Roumanie s'est confrontée à un soudain intérêt pour ses minorités ethniques. Le respect de leur droit devient dès 1993, pour l'Union européenne, l'un des critères pour juger des Etats candidats à l'adhésion (Nacu, 2009, 198). Les campagnes de communication publique, les plans anti-pauvreté et les programmes pilotes destinés aux populations romi se multiplient alors. Elles sont le fait du gouvernement roumain. Lors de cette même décennie, des crimes ou des délits pour lesquels l'ethnonyme ţigan est utilisé apparaissent dans la presse (Per, 1996). Avec le soutien du Project on Ethnic Relations, des organisations politiques romi reprochent cet usage aux médias. Des suggestions sont alors faites pour lutter contre ces travers en recrutant des jeunes romi dans les instances médiatiques et en accordant une plus grande visibilité à "l'ethnie Rom », en dehors du topos de la criminalité. Au gré d'une conjoncture alliant misérabilisme et incrimination, la "question Rom » suscite d'autres discours émanant des instances et des leaders romi. L'imprécision des logiques fondatrices des ONG romi et leurs problèmes de financement ont progressivement rendu plus de 300 associations parfaitement invisibles (Rostas, 2009, 61). A ces premières voix dissonantes, s'ajoutent celles de certains leaders de la communauté, dont les « couronnements » sous le titre de " Roi international de tous les Tsiganes » ont défrayé la chronique au cours des années 2000 (Fosztó, 2009). Leur discours et l'imagerie archaïque qu'ils ont mobilisée ont participé à la construction d'une identité diasporique imaginaire. Sur un autre plan, la reprise massive des rapports des agences de presse internationales par les médias roumains lors des expulsions de Romi en 2010 a entretenu ce même imaginaire d'une nation diasporique désaffiliée de tout état (Chiru Dumitru, 2012).

Depuis la fin des années 1990, les personnages tsiganes font leur apparition dans le cinéma et les séries télévisées en Roumanie. C’est un étranger, le réalisateur français 
Tony Gatlif, qui place, le premier, des personnages (et acteurs) ţigani au centre de son récit. En rupture avec le rôle du deutéragoniste du héros national, auquel étaient cantonnés les personnages ţigani sous l'ère communiste (Larcher, à paraître 2015), Gadjo Dilo (Gatlif, 1998) ouvre la voie aux fictions qui représenteront des figures tsiganes au cours de la décennie suivante. Le film roumain Furia (La Rage, 2002) de Radu Muntean, qui remploie certains décors et acteurs du film de Tony Gatlif, ou encore la série roumaine Băieţi buni (Les bons garçons, 2005), utilisent les conventions génériques du thriller et construisent un récit autour de personnages appartenant à une jeunesse sous-diplômée et urbaine. Le film Trei fraţi de belea (Trois frères du tonnerre, 2005) poursuit une seconde voie plus proche de la comédie et des ţigani aux costumes typés. Ce sont ces dernières figures et conventions génériques qui rencontreront d'abord un certain succès à la télévision roumaine.

Les arcs narratifs des telenovelas roumaines Inimă de ţigan (Coeur de tsigane, 2007) et Regina (La Reine, 2008) sont construits autour des alliances nouées entre plusieurs familles. Le "pilote» raconte le coup de foudre entre Irina, une Roumaine, et Codruţ, un tsigane issu d'une şatra, une communauté vivant en caravane selon une tradition fantasmée par les scénaristes de la série. Les difficultés arrivent lorsque les deux amoureux rencontrent l'opposition de leurs parents qui cherchent à les marier à des proches de leur famille respective. Les marqueurs de la frontière ethnique sont présents dans les développements du récit, les dialogues et la plastique des personnages.

Dans le respect des conventions du mélodrame, les deux premières saisons présentent des personnages secondaires regroupés en fonction de leur parenté. Les alliances et mariages, toujours problématiques dans ce genre de fictions, sont à l'origine des conflits qui se développent au cours du récit. Les dialogues et récits font donc grand usage du nom et du sang qui sont ici les matrices de la dramatisation des rapports de filiation et d'alliance. Ces discours attribuent ainsi une identité à une communauté par ailleurs inexistante à l'image ; les figurants de la şatra - issus de familles romi sont en effet cantonnés aux marges de l'image.

Devant l'altérité de cette communauté, les scénaristes ont élaboré des " personnages pédagogues » (Villez, 2014) et des "personnages délégués » (Jost, 2011, 27) qui ponctuent le récit de leur expertise sur l'intégration de la şatra au sein de la modernité urbaine. Cristofor, le consigliere roumain du chef tsigane, se pose en véritable ethnologue: «tu es tsigane, et dans tes veines coulent l'amour et la passion de la vie» (Épisode 35). Le docteur Toma est au contraire un garant de la société civile: «sur le lieu de travail, seules comptent les compétences professionnelles !»

Les ressorts qui fondent ces deux premières saisons leur font prendre parti pour les unions mixtes ou colorblind. Toutefois, si le récit tient un discours sur les unions possibles par delà les différences ethniques, ces alliances prennent davantage la forme d'adoptions et de travestissements. Ainsi la belle affublée en ţigancă n'est jamais incarnée par un personnage tsigane. La figure paradoxale de cette roumaine déguisée incarne plus une adoption qu'une alliance. Ces unions impossibles entre deux com- 
munautés sont aussi figurées par une partition chromatique qui surenchérit celle de l'appartenance ethnique des personnages ; la ţigancă brune est grotesque et les belles ţigănci sont blondes.

Contrairement aux minorités visibles et à la color line des séries télévisées occidentales, le système des personnages de ces premières telenovelas se caractérise par des mascarades - exceptée Doiniţa Oancea, les acteurs ne sont pas des Romi - et des partitions chromatiques entre blondes et brunes, parures fleuries contre blouses blanches et costumes noirs. L'usage de ces artifices pour figurer des personnages tsiganes est une tradition ancienne, présente également dans le cinéma roumain. Les scénaristes et producteurs d'Inimă de ţigan, qui sont aussi tous des Gadje, ont réactivé cette tradition en se référant au film soviétique Tabor ukhodit $v$ nebo (Les Tsiganes montent au ciel, 1976). Ce film remployait déjà une culture visuelle et populaire des figures tsiganes mises en scène par le Moscow Teatr Romen, haut lieu de patrimonialisation du répertoire folklorique russe interprété par des artistes romani (Lemon, 1996). Si leurs apparences sont semblables, les ţigani de la şatra sont toutefois moins les garants d'une tradition nationale que l'incarnation d'un rejet de l'Autre dans un régime chromatique différent. Si les médias d'information ont contribué à l'uniformisation du référent rom ou ţigan, les séries télévisées des années 2007-2009 ont figuré un essentialisme ethnique tout à fait semblable. Plutôt que d'employer les topos de la pauvreté, de la criminalité et de l'étrangeté, ces émissions télévisées ont construit une économie figurative autour d'une partition chromatique entre personnages roumains et tsiganes.

\section{Concentration médiatique, dispersion et diversification des figures tsiganes}

Avec le renouvellement des séries à partir de la rentrée 2009, les figures des premières séries télévisées vont à la fois se diversifier et se disperser au sein des chaînes et des émissions. Ce mouvement est à replacer dans un paysage médiatique caractérisé par l'engagement conjoint des principaux conglomérats médiatiques dans trois types de processus. Les premières telenovelas apparaissent quand les groupes de médias bénéficient de leurs propres régies suite à une convergence verticale accrue depuis le début des années 2000. Dans un contexte où les trois groupes médias privés les plus importants en Roumanie concentrent $57 \%$ de part d'audience nationale et $90 \%$ des recettes publicitaires, les stratégies de concurrence agressive sont nombreuses ; rachat d'émissions et de franchises, counterprogramming, etc. Enfin, l'émergence des personnages ţigani dans les telenovelas s'est faite pendant les années où la télévision a obtenu ses plus importantes recettes publicitaires $(2007,2008)$ avant que les annonceurs se retirent de manière conséquente du marché télévisuel. Suite au succès rencontré par les deux premiers cycles d'Inimă de ţigan, ce sont très probablement ces 
éléments qui expliquent le succès relatif rencontré par des figures tsiganes dans des émissions de prime time entre 2009 et 2013.

Le passage du troisième cycle de la telenovela Inimă de ţigan - State de Romania (State de Roumanie) - de la chaîne consacrée aux telenovelas (Acasă TV) sur la chaîne principale (Pro TV) du groupe Media Pro en 2009 initie plusieurs changements. Le premier est une plus grande visibilité de ces personnages de tsiganes dans diverses émissions de divertissement : Serviciul Român de Comedie (Le Service roumain de Comédie, Media Pro, 2009-2010), În Puii Mei (Dans mes poussins, Intact, 2009-2011); et de séries télévisés: Moştenirea (L’Héritage, Media Pro, 2010-2011), Iubiri secrete (Les Amours secrètes, ProSiebenSat.1, 2011-...), Spitalul de demență (L'Hôpital de folie, Media Pro, 20122013).

De plus, la şatra disparaît en 2009 et les conventions mélodramatiques cèdent progressivement leur place à celles de la comédie burlesque et du sitcom. L'altérité des personnages ţigani et leur inadaptation à leur environnement sont seulement traitées dans un registre comique, et ne nécessitent donc plus d'experts ou de pédagogues.

Par ailleurs, la diégèse des premiers récits était jusqu’à présent structurée autour d'oppositions claires entre le territoire national et l' « étranger » ou le monde urbain et rural. L'univers de ces nouvelles séries rapproche au contraire les lieux et les figures. La famille titre de State Potcovaru, par exemple, assimile un ensemble de traits contradictoires jusqu'alors incarnés par différents personnages. State devient le maire ţigan d'un petit village roumain tout en demeurant le "roi des prostituées et des mendiants de Paris ». Les arcs narratifs représentent l'Occident en un pôle d'attraction et non plus seulement de perdition, comme c'était le cas jusqu'alors. Cette rencontre entre l'exotisme des ţigani de Paris et l'archaïsme villageois révèle un univers rural aussi primitif que la şatra.

Enfin, les partitions chromatiques qui organisaient l'économie figurative des premières séries s'estompent progressivement. Ainsi, les femmes ţigănci de ces différentes émissions alternent entre parures fleuries et tenues distinguées, et les travestissements touchent maintenant d'autres personnages, comme ceux issus du ProcheOrient. Cependant, il arrive encore que ce nouveau régime visuel cohabite avec l'ancien comme dans la saison 6 de Iubiri secrete (2013-2014), où le personnage de Iani est interprété par un musicien romi tandis que sa mère - une actrice roumaine parée en ţigancă - tente de rétablir un ordre chromatique bien malmené.

\section{Nouvelles figures et « circulation des médias »}

En parallèle à cette diversification des personnages tsiganes, des nouvelles figures issues des chaînes de télévision de niche et des productions amateurs apparaissent dans les télévisions mainstream. Depuis le milieu des années 2000, des chaînes diffusant des clips vidéos de muzică populară (Favorit, Etno TV) et de manele (Taraf TV, Mynele TV) ont connu une augmentation de leur audience (Urdea, 2014 ; Schiop 2011). 
Musique populaire produite exclusivement par des musiciens romi et dont les auditeurs sont aussi bien roumains que romi (Rădulescu, 2004), le manele se distingue de la muzică populară par sa propension à faire l'objet de nombreuses publications amatrices en ligne (YouTube, Hi5, Facebook). Les foyers roumains étant de plus en plus connectés à internet, le manele participe à une véritable culture musicale, visuelle et numérique des internautes en Roumanie. Ceci est à la fois perceptible lors de controverses en ligne, comme sur la page Facebook du concert du manelist Romeo Fantastick dans un club branché de Bucarest en 2014, et dans les discours médiatiques sur les pratiques émergentes des réseaux sociaux qui, par remplois d'images et glissement de sens, participent à la formation d'un imaginaire collectif de l'internet (Larcher, 2015). Entre 2009 et 2012, l'émission În Puii Mei a produit des courts métrages autour de certains archétypes et des personnages connus du grand public; parmi ces derniers, deux séries se réapproprient des images amateurs diffusées sur internet et sur les chaînes de télévision de niche.

Pour une première série, Mihai Bendeac, le producteur de l'émission, a travaillé en collaboration avec Dan Bursuc, un impresario rom connu pour son soutien apporté à de nombreux manelişti au début de leur carrière. Il a donc constitué le groupe de musiciens qui ont participé à la réalisation de reportages télévisés parodiques. Ces musiciens interviewent Florin Salam, une star du manele, ici imité par Mihai Bendeac. Empruntant pour une part au dispositif utilisé par Salam lors de ses performances, les chanteurs (de véritables musiciens romi) entonnent une série de couplets tandis que le sosie de Salam imite grossièrement ses gestes. Ces courts métrages sont très rapidement transformés, par des amateurs, en des contenus transmédias, publiés sur les plateformes numériques (YouTube). Dernièrement, c'est la société de production de Dan Bursuc qui a republié ces courts métrage en ligne. Dans le cadre de cette collaboration, des évolutions sont cependant perceptibles entre les premiers et les derniers courts métrages (2012). Les ţigani présents dans l'émission depuis plusieurs saisons disparaissent, remplacés par des manelişti. D’autres stars du manele - y compris Dan Bursuc - ont au fur et à mesure des épisodes leur propre sosie. Les vers des chanteurs, Sasha puis Bogdan Artistu', font tout autant la promotion de leurs productions que la satire de Florin Salam. Ainsi, la ligne de démarcation entre les stratégies de ces manelişti et la condescendance avec laquelle l'émission semble les traiter est difficile à tracer. Bogdan Artistu' n'ayant par ailleurs aucun mal à faire depuis des concerts avec Florin Salam, cette ambiguïté éclaire à la fois le registre parodique de l'émission, la grande concurrence qui existe entre musiciens de manele, et les dimensions ironiques inhérentes au genre (Stoichiţă, à paraître).

De la même façon, l'équipe de În Puii Mei produira entre 2009 et 2012 une série de courts-métrages autour du personnage de Fernando, interprété par Mihai Bendeac. Les clips vidéos suivent un dispositif moins élaboré ; ils présentent des scénettes grotesques inspirées par Fernando de la Caransebeş, un musicien amateur peu connu au début de l'émission, et les parodies n'ont fait l'objet d'aucune collaboration ni 
d'accord préalable avec la personne intéressée. La série se présente comme la satire d'un archétype apparu dans les commentaires sur les nouvelles images produites sur les réseaux sociaux: le cocalar. Ce terme désigne un jeune homme qui affiche sa richesse et sa sexualité de manière ostentatoire. Son émergence, ainsi que celle de son équivalent féminin, la piţipoanca, une femme légère et à la sexualité outrageuse, est intimement liée aux discours médiatiques sur l'effet du manele sur la société roumaine (Schiop, 2011). Tous ces courts métrages de l'émission sont par la suite devenus des contenus transmédias dispersés sur les plateformes numériques. La circulation de ces images ne s'arrête pas là pour autant. Depuis la fin de l'émission, Fernando de la Caransebeş a produit quelques vidéoclips (Castravetele, Nebunia din Banat, Y'auzi ya) sous la tutelle de Şuşanu, un manelist renommé. Dans l'ensemble de ces productions, Fernando reprend ironiquement à son propre compte l'ensemble des attributs du cocalar moqués par l'émission télévisée.

Ces dernières figures tsiganes véhiculées par la télévision roumaine montrent combien la télévision mainstream a pu jouer le rôle d'un « œil identitaire », marque du centre face une altérité périphérique (Stoichiţă, 2014, 41). Elles révèlent aussi leur inscription dans de nouvelles « circulations des médias » (Jenkins, Ford, Green, 2013) où les logiques top-down des producteurs sont justement anticipées et débordées par des tactiques bottom-up de nouveaux acteurs, qui dans ce cas pratiquaient déjà l'art parodique.

\section{Conclusion : un régime visuel de l'altérité en constante métamorphose}

Sur une période relativement courte, les émissions de divertissement ont proposé des figurations de l'altérité des ţigani très différentes. Ces régimes visuels interagissent avec des discours et des pratiques très diverses. Ce passage en revue, même très rapide, montre ainsi l'évolution des sources et influences qui constituent la matrice de ces représentations: des discours des médias d'information aux images issues des champs limitrophes à celui des industries culturelles. Cette histoire visuelle montre également la métamorphose des premières partitions chromatiques et leurs identités ethniques expertisables en des figures beaucoup plus syncrétiques. Si les relations de pouvoir entre l'œil identitaire des télévisions mainstream et l'altérité des ţigani sont remises en cause par ces nouvelles circulations des médias, elles alimentent cette association entre ţigani et manele, qui est l'ultime incarnation de « la haine sociale » entretenue par la société roumaine à l'encontre des Romi/ Ţigani (Mihăilescu, 2014).

Depuis deux ans, des altérités parodiques apparaissent dans les dernières productions visuelles des manelişti ; l'Afrique noire (Bogdan Artistu, Am poftă de tine) les favelas brésiliennes, (Bogdan Artistu, Kana Jambe) et le Proche-Orient (Leo de Vis, Sistemul Seicelor, Adrian Minune, Eduard de la Roma, Dubai, Dubai). Si cette appropriation du procédé de la mascarade, par lequel les personnages ţigani ont été représentés au ci- 
néma depuis les années 1960, peut sembler en tout point réjouissante, la perspective d'une reprise de ces images par les chaînes de la télévision mainstream l'est moins.

\section{Bibliographie}

BRENEZ N. (1998), De la Figure en général et du Corps en particulier. L'invention figurative au cinéma, Bruxelles : De Boeck Université, Coll. Arts \& Cinéma.

Chiru Dumitru I. (2012), «Ego and Alter in mass media representation. Case Sudy : Journalistic Discourse on the "Expulsion of Gypsies" ». In Valentina Pricopie (coord.) Social Europe and the Media: discourses, perceptions, mentalities, București : Tritonic, 2012, p.107-117.

FoszTó L. (2009), «Transformarea sferei publice postsocialiste : o analiză a unor evenimente mass-media legate de romii din România ». In Colecţie de studii despre Romii din România, Cluj-Napoca : Editura ISPMN, Editura Kriterion, pp. 105-131.

Jenkins, Ford S., Green J., (2013), Spreadable Media. Creating Value and Meaning in a Networked Culture, New York et Londres : New York University Press.

Jost F. (2011), De quoi les séries américaines sont-elles le symptôme?, Paris: CNRS Éditions.

LARCHER J. (2015) «"Piţipoance” et figures féminines sursexualisées dans les médias roumains : intermédialité et divisions sociales ». In Dossier thématique "Le Sexe à l'Est », Regards sur l'Est, n69.

LARCHER J. (à paraître), «Le cinéma roumain au prisme de l'altérité. . Le cas des personnages tsiganes ». In Cahiers Pierre Belon / Études Balkaniques.

LEMON A. (1996), « Hot Blood and Black Pearls: Socialism, Society, and Authenticity at the Moscow Teatr Romen ». In Theatre journal, vol.48, n4, pp.479-494.

Mihailescu V. (2014), « De ce urâm Ţiganii. Eseu despre partea blestemată, manele şi manelism ». In Mihăilescu V., Matei P., Condiţia Roma şi Schimbarea discursului, Bucureşti : Polirom, pp.189-223

NACU A. (2009), «L'émergence de la “Question Rom” en Roumanie et en Bulgarie. Du consensus au stigmate ». In Tumultes, vol.1, n³2-33, pp.195-216.

Olivera M. (2009), "Introduction aux formes et raisons de la diversité Rom roumaine ». In Études Tsiganes, n³8.

Project on Ethnic Relations (1996), The Media and the Roma in Contemporary Europe: Facts and Fictions, Princeton : PER.

PIASERE L. (2004), I rom d'Europa. Una storia moderna, Roma e Bari : Laterza. 
RădulEsCu S. (2004), Taifasuri Despre Muzica Ţigănească, Bucureşti : Paideia.

RosTAS I. (2009), « Romii în România postcomunistă : mobilizare şi discurs identitar ». In Sfera Politicii, vol. XVII, n¹38, http://www.sferapoliticii.ro/sfera/138/art08rostas.html.

Schiop A. (2011), "Cum Au Îngropat Elitele României Manelele. O Poveste Cu Cocalari». In Critic Atac. $\mathrm{n}^{\circ} 2$, http://www.criticatac.ro/3957/cum-au-ingropatelitele-romaniei-manelele-o-poveste-cu-cocalari/.

STOICHiţă V.A., (à paraître), “'Yes, Yes, Yes, I'm clever and rich'. The boyar in the helicopter. Power, Parody and Carnival in Manele Performances ». In Beissinger M., Giurchescu A., Rădulescu S., ed., Manele in Romania: Cultural Expression and Social Meaning in Balkan Popular Music, Scarecrow press.

StoichițA V. I. (2014), L'image de l'Autre. Noirs, Juifs et Musulmans et « Gitans » dans l'art occidental des Temps modernes, Paris : Éditions Hazan, Louvre Éditions, La Chaire du Louvre.

URdeA A. (2014), « Folklore Music on Romanian TV. From State Socialist Television to Private Channels ». In Journal of European Television History and Culture, vol.3, $\mathrm{n}^{\circ} 5$.

VILLEZ B. (2014), Law and Order - La justice en Prime Time, Paris : PUF. 